\title{
FREE GLOBAL DSM ASSESSMENT ON LARGE SCALE AREAS EXPLOITING THE POTENTIALITIES OF THE INNOVATIVE GOOGLE EARTH ENGINE PLATFORM
}

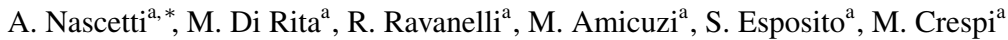 \\ ${ }^{a}$ Geodesy and Geomatics Division, DICEA, University of Rome "La Sapienza", Italy - \\ (andrea.nascetti, martina.dirita, roberta.ravanelli, mattia.crespi)@uniroma1.it
}

KEY WORDS: DSM assessment, Google Earth Engine, ASTER, SRTM, geometric accuracy

\begin{abstract}
:
The high-performance cloud-computing platform Google Earth Engine has been developed for global-scale analysis based on the Earth observation data. In particular, in this work, the geometric accuracy of the two most used nearly-global free DSMs (SRTM and ASTER) has been evaluated on the territories of four American States (Colorado, Michigan, Nevada, Utah) and one Italian Region (Trentino AltoAdige, Northern Italy) exploiting the potentiality of this platform. These are large areas characterized by different terrain morphology, land covers and slopes. The assessment has been performed using two different reference DSMs: the USGS National Elevation Dataset (NED) and a LiDAR acquisition. The DSMs accuracy has been evaluated through computation of standard statistic parameters, both at global scale (considering the whole State/Region) and in function of the terrain morphology using several slope classes. The geometric accuracy in terms of Standard deviation and NMAD, for SRTM range from 2-3 meters in the first slope class to about 45 meters in the last one, whereas for ASTER, the values range from 5-6 to 30 meters.

In general, the performed analysis shows a better accuracy for the SRTM in the flat areas whereas the ASTER GDEM is more reliable in the steep areas, where the slopes increase. These preliminary results highlight the GEE potentialities to perform DSM assessment on a global scale.
\end{abstract}

\section{INTRODUCTION}

Google Earth Engine (GEE) is a computing platform recently released by Google "for petabyte-scale scientific analysis and visualization of geospatial datasets" (Google Earth Engine Team, 2015). The GEE can be used to run geospatial analysis using a dedicated HPC (High Performance Computing) infrastructure. GEE enables researchers to access geospatial information and satellite imagery, for global and large scale remote sensing applications. The free and public data archive includes more than thirty years of historical imagery and scientific datasets, daily updated and expanded: it contains over than two petabytes of geospatial data instantly available for analysis.

The main idea behind GEE is that, also for the analysis of satellite and geospatial data, we are now moving towards the Big Data paradigm and consequently it is necessary to change the processing way from the standard procedure "bring data to users" to the opposite "bring users to data": as a matter of fact, the users can directly upload algorithms to the dedicated infrastructure removing the required time for data transfer and allowing the development of innovative applications.

The leading idea of this work is to evaluate the precision and the accuracy of two available free global Digital Surface Models (DSMs), ASTER GDEM and SRTM, on large areas, by leveraging the GEE Platform capabilities. Accordingly to previous studies (Jacobsen, K. 2013), proper routines to evaluate standard statistical parameters to represent DSM precision and accuracy (i.e. mean, median, standard deviation, NMAD, LE95) were implemented inside the GEE Code Editor. Moreover, the routines were used to characterize the accuracy of the input DSM within different slope classes.

In detail, in Section 2. a description of the GEE characteristics and functionalities is provided, paying specific attention to the

${ }^{*}$ Corresponding author capabilities it offers; Section 3. includes the data set description and Section 4. the results presentation and discussion. In the end, in Section 5., some conclusions are drawn and future prospects are outlined.

\section{GOOGLE EARTH ENGINE PLATFORM}

The continuous increase of satellite data involved a continuous demand for storage, computation capacity and efficient tools to perform geospatial analysis of such information at global scale.

The developed GEE is designed to meet these needs: as a matter of fact, it is a high-performance cloud-computing platform for global-scale Earth observation data and analysis. It enables monitoring and measurement of changes in the Earth's environment, at planetary scale, on a large catalogue of Earth observation data. Initial efforts have focused primarily on global forest monitoring and measurement in the developing world. The intent was to put this platform into the hands of scientists and developing world nations, in order to advance the broader operational deployment of existing scientific methods, and strengthen the ability for public institutions and civil society to better understand, manage and report on the state of their natural resources (Moore and Hansen, 2011).

The platform supports generation of spatial and temporal mosaics, satellite imagery composites without clouds and gaps, as well as a variety of spectral indices, and can also be expanded and modified by the user even for customized applications (Horowitz, 2015), (Pekel et al., 2016), (Donchyts et al., 2016a), (Donchyts et al., 2016b). Indeed, GEE also includes an application programming framework that allows scientists to access to computational and data resources, to scale their current algorithms or develop new ones.

The platform can be accessed by the users - upon Google approval - either by the so called Code Editor or the Explorer. The 
latter is an easy web access point to the platform - with no need of programming skills - where the user may add remote sensing datasets as well as apply some standard image analysis techniques (e.g. algebra between bands, image filters, etc.) (Agapiou, 2017). The GEE Code Editor is instead a web-based IDE for the GEE JavaScript API. Code Editor features are specifically designed to make the development of complex geospatial workflows fast and easy. Moreover, it is possible to create powerful web application using the GEE Python API, to share and widespread the scientific results obtained through the analysis performed within the platform.

\section{DATASET}

The GEE platform has been exploited in order to carry out an assessment on the free global DSMs, ASTER and SRTM. The evaluation has been performed on five different wide areas: four American States (Colorado, Michigan, Nevada, Utah) and one Italian Region (Trentino Alto-Adige, Northern Italy). The selected areas provide different land use, land covers and slopes, and are therefore suited for a comparison aimed at accuracy and reliability understanding.

For all the five analysed areas, both ASTER and SRTM have been compared to a more accurate reference DSM, that is the National Elevation Dataset (NED) for the American States, and a LiDAR DSM for the Italian region. SRTM and NED data are provided by GEE, so it is not necessary to upload them in the platform, whereas ASTER and LiDAR data have been supplied and uploaded as external input.

The Advanced Spaceborne Thermal Emission and Reflection Radiometer (ASTER), is an optical imaging instrument onboard Terra, the flagship satellite of NASA's Earth Observing System (EOS) launched in December 1999. ASTER is a cooperative effort between NASA, Japan's Ministry of Economy, Trade and Industry (METI), and Japan Space Systems. The ASTER sensor onboard of Terra satellite is designed to provide image data in 14 visible, near-infrared, short wavelength infrared and thermal infrared spectral bands. Stereo image data using both nadir and aftlooking telescopes. The freely available nearly-global ASTER GDEM, with 1 arcsec spacing (about $30 \mathrm{~m}$ ), has been generated covering the area from $83^{\circ} \mathrm{S}$ up to $83^{\circ} \mathrm{N}$ latitude. With the version 2 (GDEM2) in 2011 the horizontal location of the individual DSM have been improved, so with the GDEM2 the morphologic quality corresponds to the 1 arcsec spacing.

The Shuttle Radar Topographic Mission (SRTM) is a free nearlyglobal DSM from $56^{\circ} \mathrm{S}$ to $60^{\circ} \mathrm{N}$, produced from single-pass interferometry. As typical for SAR, the original data have gaps in mountainous areas, cities with large buildings, on water surfaces and dry sand deserts. These gaps have been filled; very often SPOT 5-HRS data have been used (Jacobsen, 2013). The elevation model is arranged into tiles, each covering one degree of latitude and one degree of longitude, named according to their south western corners. The resolution of the raw data is 1 arc-second (about $30 \mathrm{~m}$ ), but this has only been released over United States territory. For the rest of the world, only 3 arc-second (about 90 m) data are available. GEE provides the SRTM at 3 arc-second.

The NED is a seamless dataset with the best available raster elevation data of the conterminous United States, Alaska, Hawaii, and territorial islands. The NED is updated on a nominal two month cycle to integrate newly available, improved elevation source data (see Figure 1). All NED data are public domain. The NED is derived from diverse source data that are processed to a common coordinate system and unit of vertical measure. NED data are distributed in geographic coordinates in units of decimal degrees, and in conformance with the North American Datum of 1983 (NAD 83). NED data are available nationally (except for Alaska) at resolutions of 1 arc-second (about 30 meters) and 1/3 arc-second (about 10 meters), and in limited areas at 1/9 arcsecond (about 3 meters). Most NED data for Alaska are at 2 arc-second (about 60 meters) grid spacing. For the analysed areas NED is provided at $1 / 3$ arc-second.

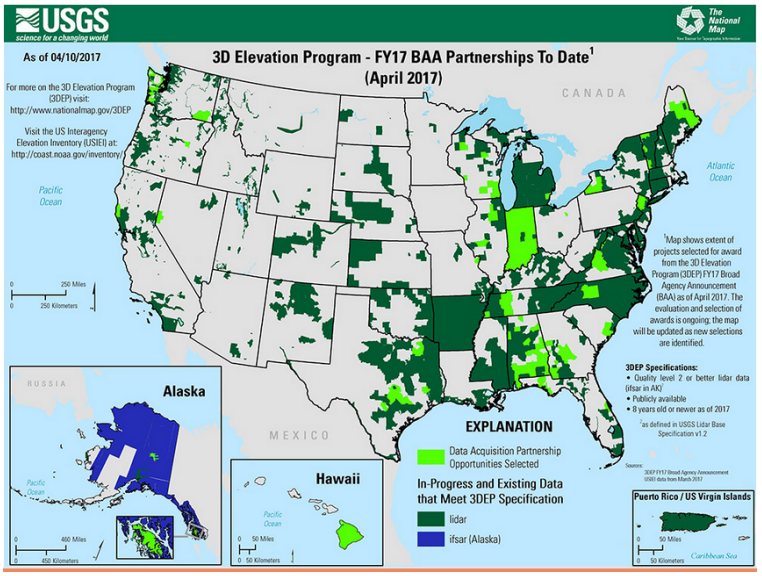

Figure 1: NED data source map

The reference DSM, obtained with LiDAR technology, exploited for the comparison, is a DSM with a grid posting $1.0 \times 1.0 \mathrm{~m}$ and a mean elevation accuracy of $0.25 \mathrm{~m}$, freely available.

\section{ASSESSMENT RESULTS}

Several studies have been conducted to evaluate the accuracy of both SRTM and ASTER DSMs, but in most of the cases the accuracy has been evaluated only on limited areas (Colmano et al., 2007), (Koch and Heipke, 2001), (Kolecka and Kozak, 2014). The main goal of this preliminary analysis is to perform a more global assessment exploiting the potentialities of GEE, and to demonstrate its capability for a nearly-global assessment of SRTM and ASTER accuracy.

In order to evaluate the DSM geometric accuracy, the height discrepancies respect to a reference DSM are computed, and subsequently the error distribution is characterized through some statistical parameters. The DSM accuracy is correlated to several factors (i.e. terrain slope, land cover) and also it depends on the data and technique used to generate the DSM (i.e. optical or SAR imagery). So the statistical conditions are not generally corresponding to a normal distribution of height discrepancies. For this reason, robust and reliable statistical parameters should be investigated in order to evaluate the DSM geometric accuracy (Jacobsen, 2013), (d'Angelo and Reinartz, 2011), (Fratarcangeli et al., 2016), (Di Rita et al., 2017).

In this work, the following most used and significant statistical parameters are considered:

- $\overline{\Delta Z}$ : mean value of the height discrepancies $\Delta Z$

- $\widetilde{\Delta Z}$ : median value of the height discrepancies $\Delta Z$

- Std.Dev: standard deviation of the height discrepancies $\Delta Z$ Std.Dev $=\sqrt{\frac{1}{N} \sum_{i=1}^{N}\left(\Delta Z_{i}-\overline{\Delta Z}\right)^{2}}$ 

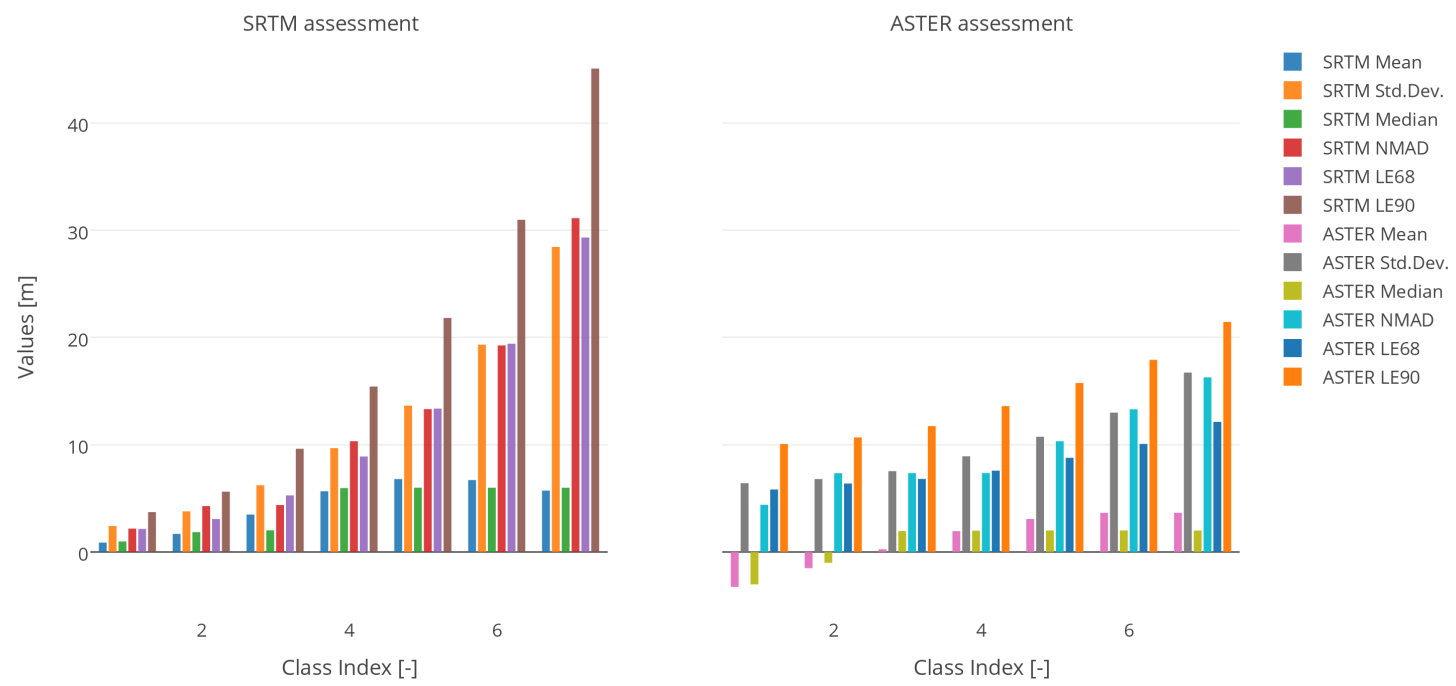

Figure 2: Colorado: SRTM and ASTER assessment results

- NMAD: Normalized Median Deviation

$N M A D=1.4826 *$ median $(|\Delta Z-\widetilde{\Delta Z}|)$

- LE68: Linear error with $68 \%$ of probability

- LE90: Linear error with $90 \%$ of probability

Exploiting the potentialities of the GEE platform for each one of the described areas, the assessment procedure has been performed. The developed code is free and open source and it is available online (Google Earth Engine DSM assessment script, 2017).

During the work, it has been noticed that the GEE percentile function is not suitable for the proper computation of the Median, NMAD, LE68 and LE90 parameters. A big discrepancy, not justified by the actual data, between the mean and the median was reported. This was probably due to the fact that, as declared in GEE documentation (Google Earth Engine Team, 2015), for small numbers of inputs the percentiles is computed directly, otherwise, for larger numbers of inputs the percentiles is derived from a histogram. The second approach could introduce significant approximation error due to the necessary linear interpolation between the bins. To overcome this issue, the percentile function of the python Numpy library (Numpy documentation, 2017) has been used to compute the Median, NMAD, LE68 and LE90 values. The Mean and the Standard deviation are computed directly using the build-in function of the GEE platform.

In order to characterize the accuracy in function of the terrain slope, the statistical parameters have been computed considering the slope classes reported in Table 1.

Table 1: Slope classes

\begin{tabular}{lccccccccc}
\hline ID class & {$[-]$} & 0 & 1 & 2 & 3 & 4 & 5 & 6 & 7 \\
\hline min slope & {$\left[^{\circ}\right]$} & 0 & 1 & 3 & 5 & 10 & 15 & 25 & 35 \\
max slope & {$\left[{ }^{\circ}\right]$} & 1 & 3 & 5 & 10 & 15 & 25 & 35 & 90 \\
\hline
\end{tabular}

In Figure 2 all the statistical parameters computed for the Colorado area are reported, where the different trends of SRTM and ASTER accuracy are reported. For the sake of brevity, for the

\begin{abstract}
Nevada: https://plot.ly/ Geod-Geom/17.embed Colorado: https://plot.1y/ Geod-Geom/19. embed Utah: $\quad$ https://plot.1y/ Geod-Geom/21.embed Michigan: https://plot.1y/ Geod-Geom/23.embed Trentino: https://plot.ly/ Geod-Geom/25.embed
\end{abstract}

other areas the results are not reported here, but all the interactive graphs are available online at the following links:

Starting from the results obtained for each area, a deeper analysis has been computed and for each slope class the mean and standard deviation of all the statistical parameters have been computed and reported in the Figures 3, 4, 6, 5, 7, 8 .

In Figures 3 and 4 a good agreement between mean and median values for both ASTER and SRTM highlights that there is no a relevant percentage of outliers in the error distributions. As regards SRTM, the mean and median values are positive in all the slope classes (close to zero in the first one). Conversely, the ASTER mean and median values are negative in the first two slope classes; this could be probably due to the elevation values used for fill the areas with water basins. A slight increasing trend of the values with respect to the slope is reported for both DSMs.

In Figures 5 and 6 Std.Dev. and NMAD values are reported. For SRTM the values of NMAD and Std.Dev. range from 2-3 meters in the first class to about 45 meters in the last one, with quite similar values between NMAD and Std.Dev., highlighting that there are few outliers in the error distributions. As regards ASTER, the values range from 5-6 to 30 meters and in the higher slope classes the gap between NMAD and Std.Dev. increases showing a higher number of outliers.

In Figures 7 and 8, the linear error values with a probability of $68 \%$ and $90 \%$ are shown. The same trend of the NMAD and Std.Dev. graph is reported.

The interactive graphs of all these parameters are also available online at the following links:

Overall, the results highlight that the accuracy decreases with the increase of the slope, and this trend is visible in all the considered parameters. Regarding the small dispersion of the values within the different slope classes, the trend appears robust both 

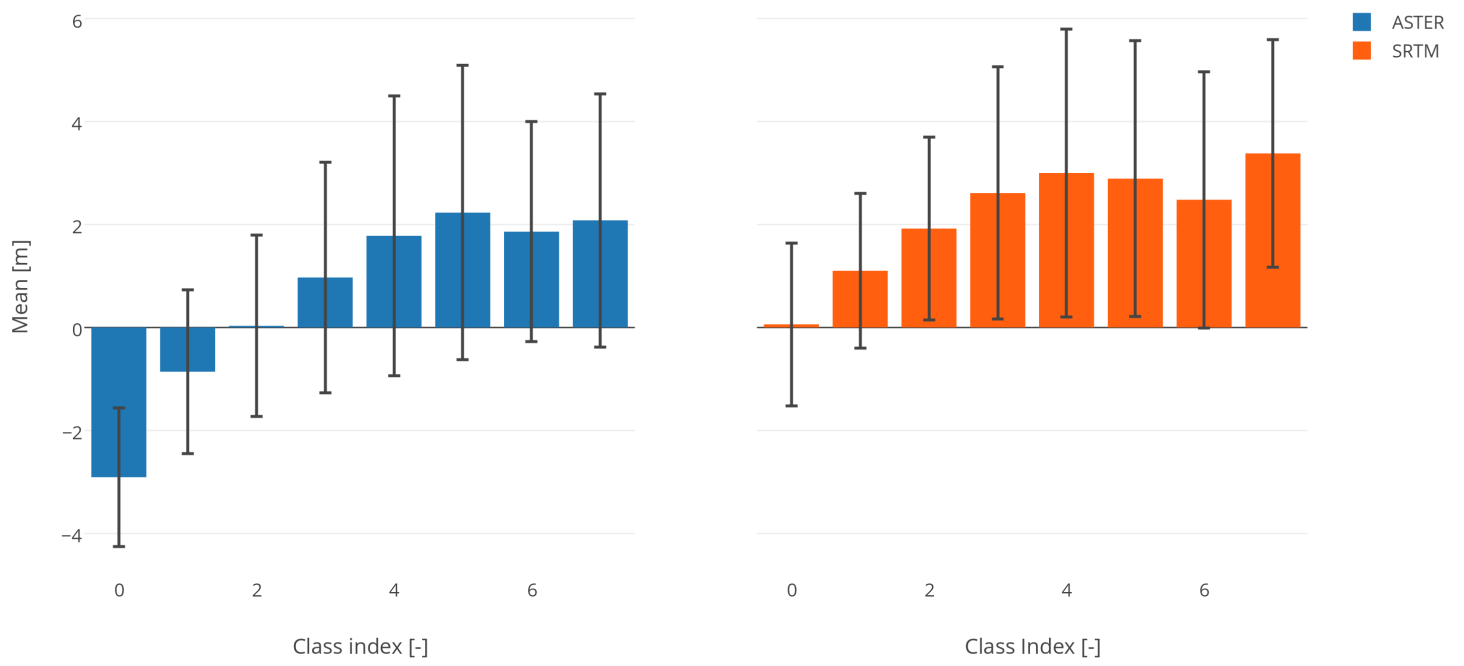

Figure 3: SRTM vs ASTER Mean analysis
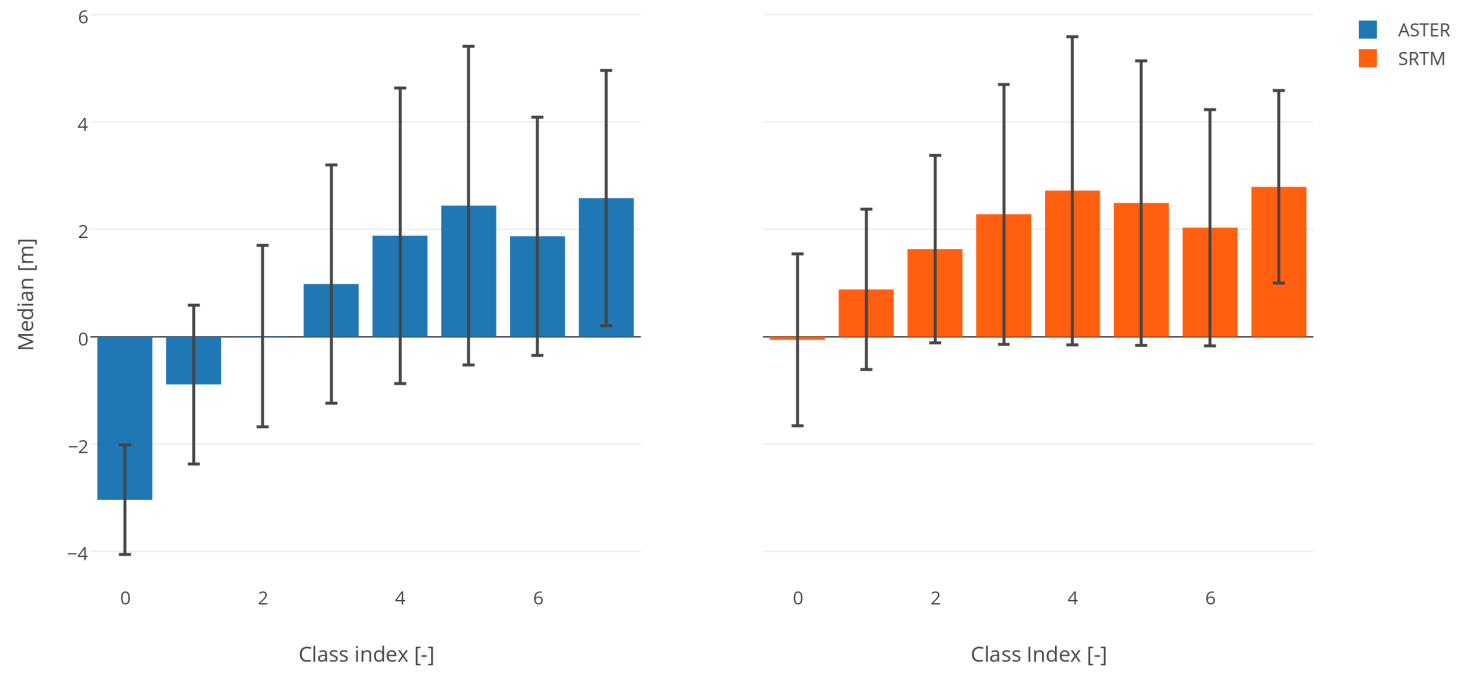

Figure 4: SRTM vs ASTER Median analysis

\begin{abstract}
Mean: $\quad$ https://plot.1y/ Geod-Geom/1.embed
Median: https://plot.ly/ Geod-Geom/11.embed

Std.Dev: https://plot.ly/ Geod-Geom/5.embed

NMAD: https://plot.1y/ Geod-Geom/13.embed

LE68: $\quad$ https://plot.1y/ Geod-Geom/9.embed

LE90: $\quad$ https://plot.ly/ Geod-Geom/15. embed
\end{abstract}

for ASTER and SRTM. Moreover, a better accuracy for the first four classes is shown in SRTM DSM with respect to ASTER; for higher slopes the SRTM accuracy decreases quickly and ASTER performs better. This behaviour is due to the different nature of the data and techniques used to generate the two DSMs (see Section 3.), and does suggest a model to take full advantage from both of them. ASTER is generated with optical stereo imagery, whereas SRTM uses InSAR data, that are affected by large distortion in mountainous areas where the slopes are higher (i.e. layover, foreshortening) (Nascetti et al., 2015).

\section{CONCLUSIONS}

GEE is high-performance cloud-computing platform for globalscale Earth observation data and analysis that has shown is capabilities in different applications.

Here the results obtained exploiting GEE for DSMs accuracy assessment on large scale areas are reported. An open source routine has been developed and used for SRTM and ASTER DSMs assessment on four American States and one Italian Region.

The outcomes highlight a good agreement among the statistical parameters, at a global level for each wide areas analysed, and within the single slope class: both mean and median, and Std.Dev. and NMAD are consistent between them.

Overall, all the results achieved are pretty consistent showing a good accordance in their behaviour: SRTM and ASTER achieve almost the same results when compared both to NED and to LiDAR. In particular, the accuracies decrease with the increase of 


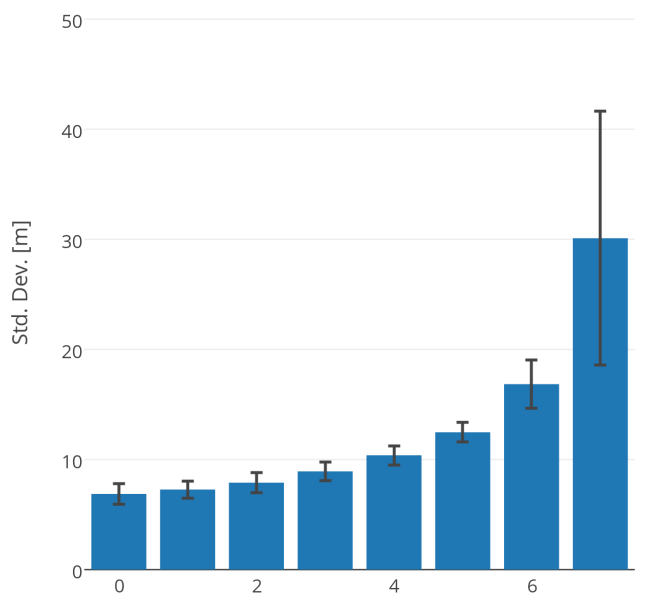

Class index [-]

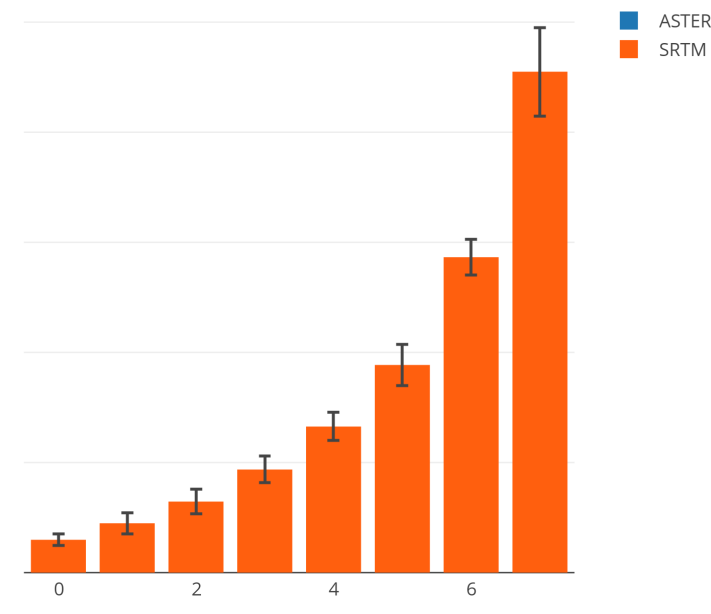

Class Index [-]

Figure 5: SRTM vs ASTER Std.Dev. analysis
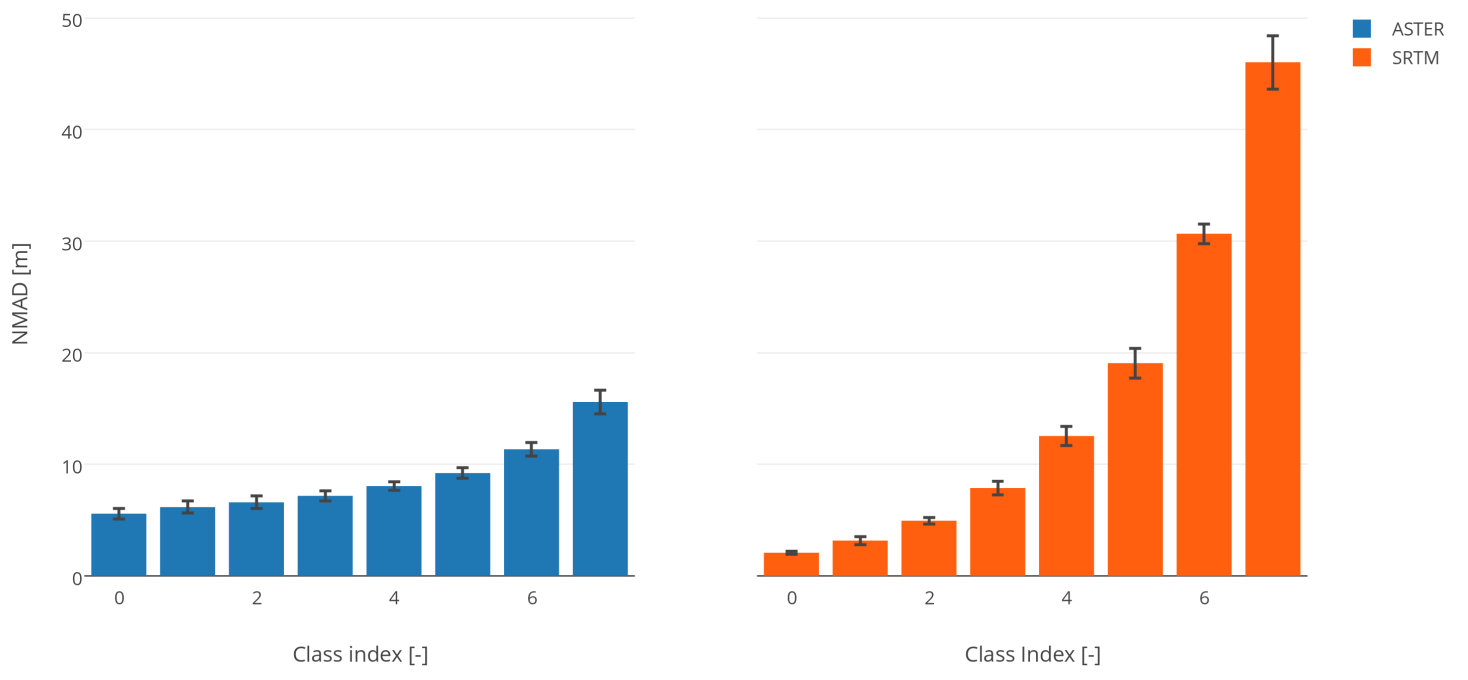

Figure 6: SRTM vs ASTER NMAD analysis

the slopes, with better results generated with SRTM for the first classes and, instead, a better behaviour shown by ASTER for the higher classes. This is due essentially to the different nature of the two DSMs (SRTM is SAR-based, ASTER is optical-based) and it could lead to make some assumptions about an optimum free nearly-global DSM: starting from the knowledge of the slope classes where they present a better accuracy with respect to the other, a more accurate global DSM can be generated as a result of an integration of both information. Therefore, it would be worthy considering the identification of a model that could describe this optimal model based on the slopes of the areas analysed.

\section{ACKNOWLEDGEMENTS}

The authors are indebted with Dr. David Colmano from "Provincia Autonoma di Bolzano", for having kindly provided the LiDAR data.

\section{REFERENCES}

Agapiou, A., 2017. Remote sensing heritage in a petabyte-scale: satellite data and heritage Earth Engine (c) applications. International Journal of Digital Earth 10(1), pp. 85-102.

Colmano, D., Crespi, M., Fabiani, U. and Zebisch, M., 2007. Quality assessment of commercially available DEMs in mountain areas. Geologic Hazards in Mountainous Areas.

d'Angelo, P. and Reinartz, P., 2011. Semiglobal Matching Results on the Isprs Stereo Matching Benchmark. ISPRSInternational Archives of the Photogrammetry, Remote Sensing and Spatial Information Sciences 3819, pp. 79-84.

Di Rita, M., Nascetti, A. and Crespi, M., 2017. Open source tool for dsms generation from high resolution optical satellite imagery: development and testing of an ossim plug-in. International Journal of Remote Sensing 38(7), pp. 1788-1808.

Donchyts, G., Baart, F., Winsemius, H., Gorelick, N., Kwadijk, J. 


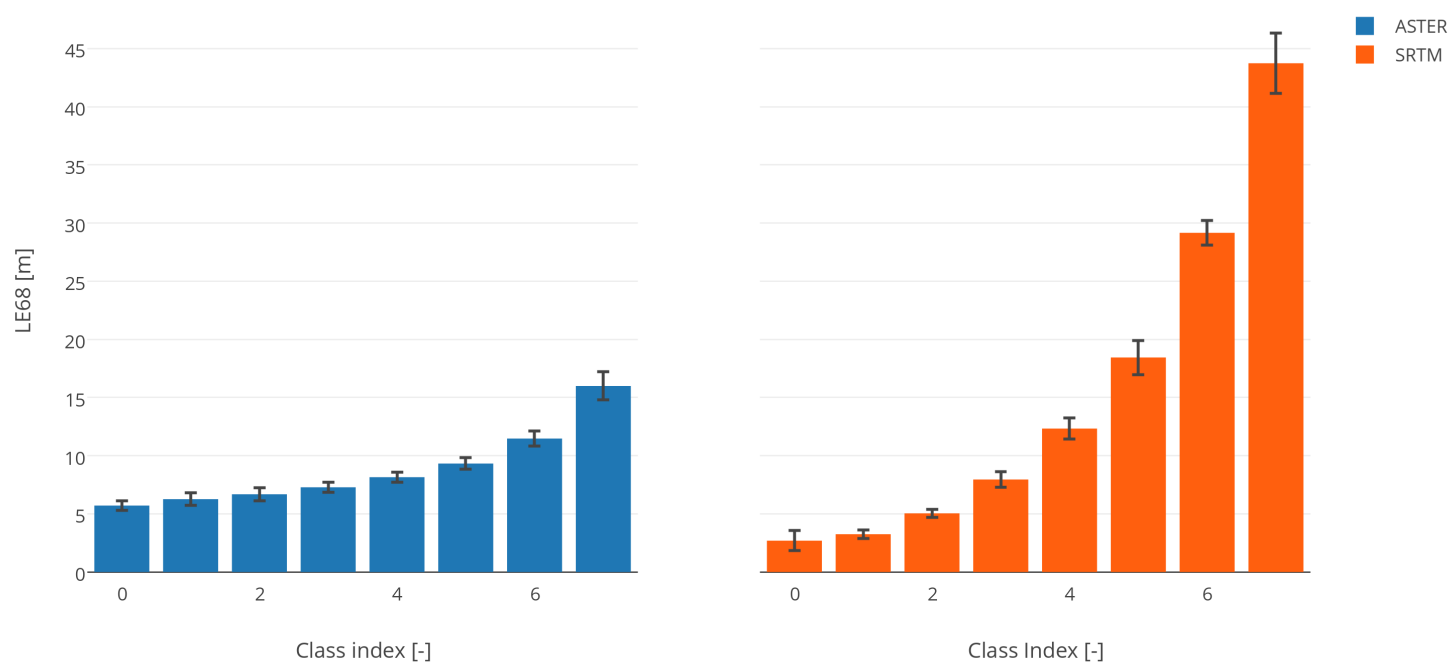

Figure 7: SRTM vs ASTER LE68 analysis

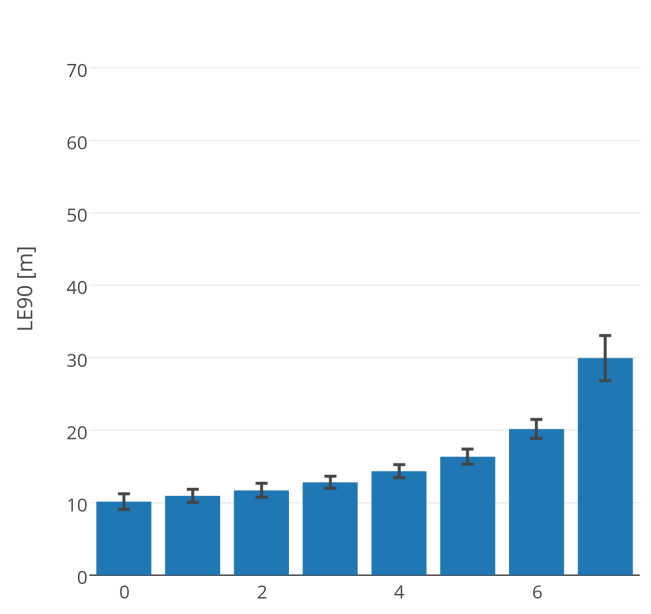

Class index [-]

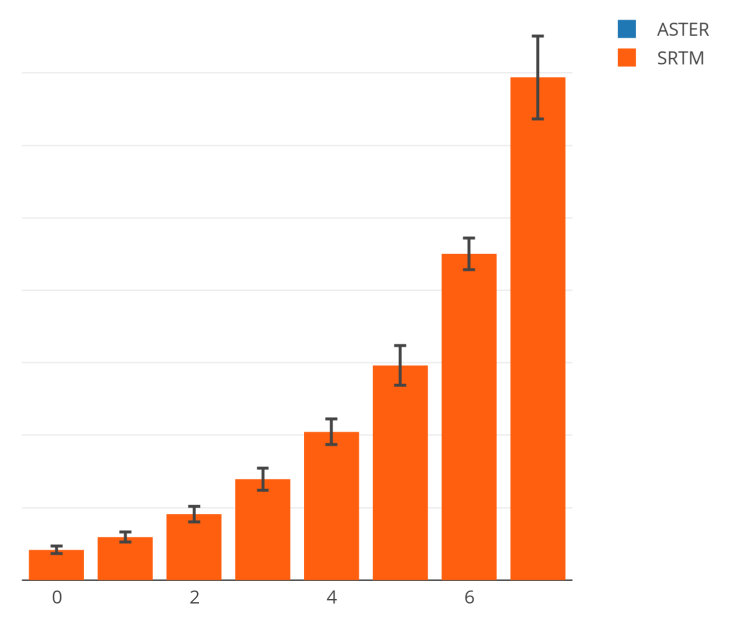

Class Index [-]

Figure 8: SRTM vs ASTER LE90 analysis

and van de Giesen, N., 2016a. Earth's surface water change over the past 30 years. Nature Climate Change 6(9), pp. 810-813.

Donchyts, G., Schellekens, J., Winsemius, H., Eisemann, E. and van de Giesen, N., 2016b. A 30 m Resolution Surface Water Mask Including Estimation of Positional and Thematic Differences Using Landsat 8, SRTM and OpenStreetMap: A Case Study in the Murray-Darling Basin, Australia. Remote Sensing 8(5), pp. 386.

Fratarcangeli, F., Murchio, G., Di Rita, M., Nascetti, A. and Capaldo, P., 2016. Digital surface models from ziyuan-3 triplet: performance evaluation and accuracy assessment. International Journal of Remote Sensing 37(15), pp. 3505-3531. cited By 2.

Google Earth Engine DSM assessment script, $2017 . \quad$ https://code.earthengine.google.com/ 27af82bbf 9be1503f 4f65e7a0113ba9a.

Google Earth Engine Team, 2015. Google Earth Engine: A Planetary-scale Geospatial Analysis Platform. https:// earthengine-google.com.
Horowitz, F. G., 2015. MODIS Daily Land Surface Temperature Estimates in Google Earth Engine as an Aid in Geothermal Energy Siting. In: Proceedings World Geothermal Congress.

Jacobsen, K., 2013. Characteristics and accuracy of large area covering height models. ISPRS-International Archives of the Photogrammetry, Remote Sensing and Spatial Information Sciences 1(1), pp. 157-162.

Koch, A. and Heipke, C., 2001. Quality assessment of digital surface models derived from the shuttle radar topography mission (srtm). Vol. 6, pp. 2863-2865.

Kolecka, N. and Kozak, J., 2014. Assessment of the accuracy of srtm c- and x-band high mountain elevation data: a case study of the polish tatra mountains. Pure and Applied Geophysics 171(6), pp. 897-912.

Moore, R. T. and Hansen, M. C., 2011. Google Earth Engine: a new cloud-computing platform for global-scale earth observation data and analysis. In: American Geophysical Union, Fall Meeting, Vol. 1, p. 02. 
Nascetti, A., Capaldo, P., Porfiri, M., Pieralice, F., Fratarcangeli, F., Benenati, L. and Crespi, M., 2015. Fast terrain modelling for hydrogeological risk mapping and emergency management: the contribution of high-resolution satellite sar imagery. Geomatics, Natural Hazards and Risk 6(5-7), pp. 554-582. cited By 7.

Numpy documentation, 2017. https://docs.scipy.org/ doc/numpy/reference/.

Pekel, J.-F., Cottam, A., Gorelick, N. and Belward, A. S., 2016.

High-resolution mapping of global surface water and its longterm changes. Nature 540(7633), pp. 418-422. 